\title{
PSEUDO-RIEMANNIAN WEAKLY SYMMETRIC MANIFOLDS
}

\author{
ZHIQI CHEN AND JOSEPH A. WOLF
}

\begin{abstract}
There is a well developed theory of weakly symmetric Riemannian manifolds. Here it is shown that several results in the Riemannian case are also valid for weakly symmetric pseudo-Riemannian manifolds, but some require additional hypotheses. The topics discussed are homogeneity, geodesic completeness, the geodesic orbit property, weak symmetries, and the structure of the nilradical of the isometry group. Also, we give a number of examples of weakly symmetric pseudo-Riemannian manifolds, some mirroring the Riemannian case and some indicating the problems in extending Riemannian results to weakly symmetric pseudoRiemannian spaces.
\end{abstract}

\section{INTRODUCTION}

There have been several important extensions of the theory of Riemannian symmetric spaces. Weakly symmetric spaces, introduced by A. Selberg [10, play key roles in number theory, Riemannian geometry and harmonic analysis. Pseudo-Riemannian symmetric spaces, including semisimple symmetric spaces, play central but complementary roles in number theory, differential geometry and relativity, Lie group representation theory and harmonic analysis. Here we study the common extension of these two branches of symmetric space theory, that of weakly symmetric pseudo-Riemannian manifolds.

It is surprising that the theory of weakly symmetric pseudo-Riemannian manifolds has so many open problems. Here we recall what is known in its differential-geometric aspect and prove a few new results. Some facts, such as homogeneity for weakly symmetric pseudo-Riemannian spaces, are easy. Others, in particular questions of sectional curvature and the structure of the nilradical of the isometry group, are subtle.

In Section 1 we review a number of basic facts on the geometry of pseudo-Riemannian manifolds, especially concerning completeness and homogeneity, pointing out the contrast with the Riemannian case.

Section 2 specializes to the setting of weakly symmetric pseudo-Riemannian manifolds. After the definition, we give a short proof of homogeneity in Proposition 2.2. In Proposition 2.3 we describe circumstances under which a pair of points can be interchanged by an isometry. Example 2.4 exhibits a class of symmetric (thus weakly symmetric) pseudo-Riemannian manifolds in which not every pair of points is joined by an unbroken geodesic. We then turn to weak symmetries and weakly symmetric coset spaces. In Proposition 2.6 we recall their relation to weakly symmetric Riemannian manifolds, and in Proposition 2.7 we extend this to the setting of weakly symmetric pseudo-Riemannian manifolds.

Section 3 presents a number of examples of weakly symmetric pseudo-Riemannian manifolds related in one way or another to weakly symmetric Riemannian manifolds.

In Section 4 we study geodesics and prove the geodesic orbit property for weakly symmetric pseudo-Riemannian manifolds. We note that the fact that the property that the nilradical of the isometry group is 2-step nilpotent, which holds in the Riemannian case, fails in the pseudoRiemannian setting. Then we give a condition under which it holds for weakly symmetric pseudo-Riemannian manifolds. 


\section{Pseudo-Riemannian manifolds}

We collect some basic facts on pseudo-Riemannian manifolds, following [8].

Definition 1.1. A pseudo-Riemannian manifold $(M,\langle\rangle$,$) is a smooth manifold M$ with a nondegenerate inner product $\langle$,$\rangle on the fibers of its tangent bundle T M$. Let the expression $\left(n_{+}, n_{-}\right)$, where $n_{+}+n_{-}=\operatorname{dim} M$, denote the signature of $\langle$,$\rangle . The manifold (M,\langle\rangle$,$) Riemannian in the$ case where $\langle$,$\rangle has signature (\operatorname{dim} M, 0)$, i.e. is positive definite.

Definition 1.2. A broken geodesic is a piecewise smooth curve segment whose smooth subsegments are geodesics. A pseudo-Riemannian manifold is said to be geodesically complete if every maximal geodesic is defined on the entire real line.

Lemma 1.3. A pseudo-Riemannian manifold is connected if and only if any two points can be connected by a broken geodesic.

In the connected Riemannian case we have a metric space structure where the distance $d(x, y)$ is the infimum of the lengths of sectionally smooth curves joining $x$ to $y$. The classical HopfRinow theorem is

Theorem 1.4. For a connected Riemannian manifold $M$, the following conditions are equivalent.

(1) As a metric space under the Riemannian metric $M$ is complete, i.e., every Cauchy sequence converges.

(2) There exists a point $x \in M$ from which $M$ is geodesically complete, i.e., exp $p_{x}$ defines on the entire tangent space $T_{x} M$.

(3) $M$ is geodesically complete.

(4) Every closed bounded subset of $M$ is compact.

Furthermore,

Proposition 1.5. If a connected Riemannian manifold is complete, then any two of its points are joined by a minimizing geodesic segment.

Definition 1.6. Let $\left(M,\langle,\rangle_{M}\right)$ and $\left(N,\langle,\rangle_{N}\right)$ be pseudo-Riemannian manifolds. An isometry from $M$ to $N$ is a diffeomorphism $\phi: M \rightarrow N$ satisfying $\langle d \phi(v), d \phi(w)\rangle_{N}=\langle v, w\rangle_{M}$ for any $v, w \in T_{x} M$ and $x \in M$.

Definition 1.7. A pseudo-Riemannian manifold $(M,\langle\rangle$,$) is homogeneous if for any points x$ and $y$ there exists an isometry $\phi$ of $M$ such that $\phi(x)=y$.

Let $I(M,\langle\rangle$,$) denote the isometry group of the pseudo-Riemannian manifold (M,\langle\rangle$,$) . Then$ $I(M,\langle\rangle$,$) , with the compact-open topology, is a Lie group. A pseudo-Riemannian manifold$ $(M,\langle\rangle$,$) is homogeneous if I(M,\langle\rangle$,$) is transitive on M$.

Lemma 1.8. A Riemannian homogeneous manifold is complete. There exist homogeneous pseudo-Riemannian manifolds that are not geodesically complete.

Together with Proposition 1.5, we have:

Lemma 1.9. Any two points of a connected Riemannian homogeneous manifold is joined by a minimizing geodesic segment. This fails for some pseudo-Riemannian manifolds.

Lemma 1.10. If a Lie group acts transitively on a connected manifold, then so does the identity component of the Lie group. In particular if $(M,\langle\rangle$,$) is a connected homogeneous pseudo-$ Riemannian manifold then the identity component $I(M,\langle,\rangle)^{0}$ of its isometry group is transitive. 


\section{Geometry of PSEudo-Riemannian Weakly SymmetriC ManifoldS}

There are a number of equivalent conditions that can be taken as the definition of weak symmetry for a Riemannian manifold. The one on reversing geodesics is also appropriate in the pseudo-Riemannian case.

Definition 2.1. Let $(M,\langle\rangle$,$) be a pseudo-Riemannian manifold. Suppose that for every x \in M$ and every nonzero tangent vector $\xi \in T_{x} M$, there is an isometry $\phi=\phi_{x, \xi}$ of $(M,\langle\rangle$,$) such that$ $\phi(x)=x$ and $d \phi(\xi)=-\xi$. Then we say that $(M,\langle\rangle$,$) is a pseudo-Riemannian weakly symmetric$ manifold. In particular, if $(M,\langle\rangle$,$) is a Riemannian manifold, then we say that (M,\langle\rangle$,$) is weakly$ symmetric.

Of course the symmetric case is the case where each $\phi_{x, \xi}$ is independent of $\xi$, in other words where for any $x \in M$ the geodesic reflection at $x$ extends to a globally defined isometry of $M$. Equivalently, $(M,\langle\rangle$,$) is a pseudo-Riemannian symmetric space if for any x \in M$ there is an involutive isometry $\theta_{x}$ of $M$ that has $x$ as an isolated fixed point.

As in the Riemannian case we have

Proposition 2.2. Let $(M,\langle\rangle$,$) be a connected pseudo-Riemannian weakly symmetric manifold.$ Then $(M,\langle\rangle$,$) is a pseudo-Riemannian homogeneous space G / H$ where $G=I(M,\langle,\rangle)^{0}$.

Proof. For any $x, y \in M$, by Lemma 1.3, there exists a broken geodesic connecting $x$ and $y$. Assume that the broken geodesic is $o_{1}^{\prime} o_{2}^{\prime} \cdots o_{p}^{\prime}$, where $o_{i}^{\prime} o_{i+1}^{\prime}$ is a geodesic segment for any $1 \leq i \leq p-1, o_{1}^{\prime}=x$ and $o_{p}^{\prime}=y$. Let $\gamma_{i}$ denote the geodesic arc from $o_{i}^{\prime}$ to $o_{i+1}^{\prime}$ parameterized from 0 to 1 proportional to the arc length for any $i$. Since $(M,\langle\rangle$,$) is weakly symmetric, we$ have that there exists $g_{i} \in I(M,\langle\rangle$,$) such that g_{i} \gamma_{i}\left(\frac{1}{2}\right)=\gamma_{i}\left(\frac{1}{2}\right)$ and $d g_{i} \gamma_{i}^{\prime}\left(\frac{1}{2}\right)=-\gamma_{i}^{\prime}\left(\frac{1}{2}\right)$. Then $g_{i}$ interchanges $o_{i}^{\prime}$ and $o_{i+1}^{\prime}$. Thus $g_{p-1} \cdots g_{1}(x)=y$. So $I(M,\langle\rangle$,$) is transitive on M$. By Lemma 1.10, $I(M,\langle,\rangle)^{0}$ acts transitively on $M$. So $(M,\langle\rangle$,$) is homogeneous.$

Let $(M,\langle\rangle$,$) be a connected Riemannian weakly symmetric manifold. It is homogeneous,$ hence complete, so any two points in $M$ are connected by a geodesic segment. Thus for any $x, y \in M$ there exists an involutive isometry (the geodesic symmetry at the midpoint of that geodesic segment) that interchanges $x$ and $y$. In the pseudo-Riemannian case, although we know that $(M,\langle\rangle$,$) is homogeneous, there might not be a geodesic joining any two points. So we only$ have

Proposition 2.3. Let $(M,\langle\rangle$,$) be a connected pseudo-Riemannian manifold. If (M,\langle\rangle$,$) is$ weakly symmetric, then for any $x, y \in M$ connected by a geodesic, there exists an isometry which interchanges $x$ and $y$. In particular if $(M,\langle\rangle$,$) is Riemannian, then for any x, y \in M$ there exists an isometry which interchanges $x$ and $y$.

Example 2.4. Here is a class of pseudo-Riemannian symmetric manifolds that have points which cannot be joined by a single geodesic. Let $G$ be a semisimple Lie group such that the exponential map exp $: \operatorname{Lie}(G) \rightarrow G$ is not surjective, and let $x$ be a point of $G$ that is not in the image of $\exp : \operatorname{Lie}(G) \rightarrow G$. Such Lie groups exist, for example if $G=S L(3 ; R)$, see [2]. Use the Killing form for the pseudo-Riemannian metric. Then $G$ should be a pseudo-Riemannian symmetric space, where the symmetry at the identity element is $g \mapsto g^{-1}$, and the geodesics are the group translates of the orbits of one-parameter subgroups. See Theorem 4.2 below. This shows that there is no geodesic from the identity element to the point $x$.

Definition 2.5. Let $G$ be a connected Lie group and $H$ be a closed subgroup. Suppose that $\sigma$ is an automorphism of $G$ such that $\sigma(g) \in H g^{-1} H$ for every $g \in G$. Then we say that $G / H$ is a weakly symmetric coset space, that $(G, H)$ is a weakly symmetric pair, and that $\sigma$ is a weak symmetry of $G / H$. 
Consider the case where $H$ is a compact subgroup. The next proposition recalls the relation between group-theoretic notion and differential-geometric notions of weak symmetry. There the condition of $g(x)=\phi(y)$ and $g(y)=\phi(x)$ corresponds to the interchange condition between pairs of points.

Proposition 2.6 ([11]). Let $G$ be a connected Lie group, $K$ be a compact subgroup and $M=$ $G / K$.

(1) $G / K$ is a weakly symmetric coset space if and only if there is a diffeomorphism $\phi$ of $M$ such that $\phi G \phi^{-1}=G$ and if $x, y \in M$ there exists $g \in G$ with $g(x)=\phi(y)$ and $g(y)=\phi(x)$.

(2) Assume that the above equivalent conditions hold. Then every G-invariant Riemannian metric on $M$ is $\phi$-invariant and weakly symmetric. In particular there exists a G-invariant $\phi$-invariant Riemannian metric on $M$.

More generally, we have

Proposition 2.7. Let $G$ be a connected Lie group, $H$ be a closed subgroup and $M=G / H$.

(1) If there is a diffeomorphism $\phi$ of $M$ such that $\phi G \phi^{-1}=G$ and if $x, y \in M$ there exists $g \in G$ with $g(x)=\phi(y)$ and $g(y)=\phi(x)$, then $G / H$ is a weakly symmetric coset space.

(2) If $G / H$ is a weakly symmetric coset space, then there is a diffeomorphism $\phi$ of $M$ such that $\phi G \phi^{-1}=G$ and if $x, y \in M$ connecting by a geodesic there exists $g \in G$ with $g(x)=\phi(y)$ and $g(y)=\phi(x)$. Moreover, every $G$-invariant pseudo-Riemannian metric on $M$ is $\phi$-invariant and weakly symmetric.

(3) If there is a diffeomorphism $\phi$ of $M$ such that $\phi G \phi^{-1}=G$ and if $x, y \in M$ connecting by a geodesic there exists $g \in G$ with $g(x)=\phi(y)$ and $g(y)=\phi(x)$, then for every $g \in G$ there is $h \in G$ and an automorphism $\sigma$ of $G$ such that $\sigma\left(h^{-1} g h\right) \in H h^{-1} g^{-1} h H$.

Proof. The first two assertions follow from the proof for the Riemannian case. For the third one, assume that there exists a diffeomorphism $\phi$ such that the conditions of (3) hold. Translating by an element of $G$ we may assume that $\phi(1 H)=1 H$. Then $\sigma: g \mapsto \phi g \phi^{-1}$ defines an automorphism $\sigma$ of $G$ such that $\sigma(H)=H$ and $\phi(g H)=\sigma(g) H$. Fix $g \in G$ and $g \notin H$. Let $g^{\prime} H$ be a fixed point of $g$ in $G / H$ and $U$ be a normal neighborhood of $g^{\prime} H$. Then $U \cap g U$ is a neighborhood of $g^{\prime} H$. Then there exists $g_{1} H \in U$ such that $g_{1} H \neq g g_{1} H$ and $g\left(g_{1} H\right) \in U \cap g U$. That is, there is a geodesic connecting $g_{1} H$ and $g\left(g_{1} H\right)$. Then $1 H$ and $g_{1}^{-1} g g_{1} H$ are connected by a geodesic. By the assumption, there exists $g_{2} \in G$ such that

$$
g_{2} g_{1}^{-1} g g_{1} H=s(1 H)=1 H \text { and } g_{2}(1 H)=s\left(g_{1}^{-1} g g_{1} H\right) .
$$

It follows that $\sigma\left(g_{1}^{-1} g g_{1}\right) \in H g_{1}^{-1} g^{-1} g_{1} H$.

\section{EXAMPLES OF PSEUDO-RIEMANNIAN WEAKLY SYMMETRIC SPACES}

We describe a number of weakly symmetric pseudo-Riemannian manifolds. The reductive ones will be familiar to many readers.

Example 3.1. $M_{p, q}=G_{p, q ; \mathbb{C}} / U(p, q ; \mathbb{C})$ where $G_{p, q ; \mathbb{C}}$ is given as follows. The space $\mathbb{C}^{n}$ of $n$ tuples over $\mathbb{C}$ is viewed as a right vector space (so that it is easy to extend considerations from $\mathbb{C}$ to the quaternions and the octonions). Scalars act on the right and linear transformations act on the left. For any non-negative integers $p$ and $q$ and $n=p+q$, we have the Hermitian vector space $\mathbb{C}^{p, q}$ with the Hermitian form

$$
\langle v, w\rangle_{1}=\sum_{i=1}^{p} v^{i} \bar{w}^{i}-\sum_{i=1}^{q} v^{p+i} \bar{w}^{p+i} .
$$

Its unitary group is $U(p, q ; \mathbb{C})$. We have a Heisenberg group $H_{p, q ; \mathbb{C}}$ which is the real vector space $\operatorname{Im} \mathbb{C}+\mathbb{C}^{p, q}$ with the group composition

$$
(v, w)\left(v^{\prime}, w^{\prime}\right)=\left(v+v^{\prime}+\operatorname{Im} h\left(w, w^{\prime}\right), w+w^{\prime}\right) .
$$


Then $g(v, w)=(v, g(w))$ defines an action of the unitary group $U(p, q ; \mathbb{C})$ by automorphisms on $H_{p, q ; \mathbb{C}}$. The semidirect product group $G_{p, q ; \mathbb{C}}:=H_{p, q ; \mathbb{C}} \rtimes U(p, q ; \mathbb{C})$ has group composition

$$
(v, w, g)\left(v^{\prime}, w^{\prime}, g^{\prime}\right)=\left(v+v^{\prime}+\operatorname{Im} h\left(w, g\left(w^{\prime}\right)\right), w+g\left(w^{\prime}\right), g g^{\prime}\right) .
$$

It is clear that $H_{p, q ; \mathbb{C}}$ is 2 -step nilpotent. If $q=0$, denote $H_{n, 0 ; \mathbb{C}}$ and $G_{n, 0 ; \mathbb{C}}$ by $H_{n ; \mathbb{C}}$ and $G_{n ; \mathbb{C}}$ respectively. The usual Heisenberg group $H_{\mathbb{C}}$ is $H_{n ; \mathbb{C}}$. For details and extension to quaternionic and octonionic Heisenberg groups see [11].

The action of $G_{p, q ; \mathbb{C}}$ on $M_{p, q}:=G_{p, q ; \mathbb{C}} / U(p, q ; \mathbb{C})$ is transitive. At $x:=(1,0, \cdots, 0)$ the isotropy subgroup of $G_{p, q ; \mathbb{C}}$ is $U(p, q ; \mathbb{C})$. The tangent space at $x$ can be viewed as $\operatorname{Im} \mathbb{C}+\mathbb{C}^{p, q}$, and $d g(v, w)=(v, g w)$ for any $(v, w) \in T_{x} M_{p, q}$ and $g \in U(p, q ; \mathbb{C})$. Any $G_{p, q ; \mathbb{C} \text {-invariant pseudo- }}$ Riemannian metric is determined by a $U(p, q ; \mathbb{C})$-invariant inner product on $T_{x} M_{p, q}$. The action of $U(p, q ; \mathbb{C})$ on $\mathbb{C}^{p, q}$ is absolutely irreducible, and it is and trivial on $\operatorname{Im} \mathbb{C}$, so every $U(p, q ; \mathbb{C})$ invariant inner product $\langle$,$\rangle on T_{x} M_{p, q}$ satisfies $\left\langle\operatorname{Im} \mathbb{C}, \mathbb{C}^{p, q}\right\rangle=0$. Let $\left\langle v, v^{\prime}\right\rangle_{1}=v \bar{v}^{\prime}$, the usual inner product on $\operatorname{Im} \mathbb{C}$, and $\left\langle w, w^{\prime}\right\rangle_{2}=\operatorname{Re} h\left(w, w^{\prime}\right)$, the usual (real) inner product on $\mathbb{C}^{p, q}$. Then $\langle$,$\rangle can be any real linear combination a\langle,\rangle_{1} \oplus b\langle,\rangle_{2}, a \neq 0 \neq b$. That gives all the $G_{p, q ; \mathbb{C} \text {-invariant }}$ pseudo-Riemannian metrics on $M_{p, q}$.

The map $\phi$ given by conjugation on each coordinate is an isometry of $M_{p, q}$. More precisely,

$$
\phi(x)=x \text { and } d \phi(v, w)=(-v, \sqrt{-1} w) .
$$

Furthermore, there exists an element $g_{w}$ in $U(p, q ; \mathbb{C})$ satisfying $d g_{w}(-v, \sqrt{-1} w)=(-v,-w)$ since $U(p, q ; \mathbb{C})$ acts transitively on any cone or quadric $\left\{w \in \mathbb{C}^{p, q} \backslash\{0\} \mid h(w, w)=r\right\}$. That is, the isometry $g_{w} \cdot \phi$ satisfies

$$
g_{w} \cdot \phi(x)=x \text { and } d g_{w} \cdot d \phi(v, w)=(-v,-w) .
$$

Since the isotropy group at different points are conjugate in $G_{p, q, \mathbb{C}}$ for a homogeneous space, we have:

(1) $\left(M_{p, q},\langle\rangle,\right)$ is a pseudo-Riemannian weakly symmetric manifold.

(2) $\left(M_{p, q},\langle\rangle,\right)$ is a Riemannian weakly symmetric manifold if and only if, either $q=0$ and $a, b$ are positive, or $p=0$ and $a$ is positive and $b$ are negative.

The variation of pseudo-Riemannian weakly symmetric structure just described, is a simple group-theoretic phenomenon. Let $(M,\langle\rangle$,$) be a pseudo-Riemannian weakly symmetric manifold.$ Express $M=G / H$ where $G=I(M,\langle\rangle$,$) . Then, from the action of the isotropy subgroup H$ on the tangent space to $M$ at $1 H$ we see that every $G$-invariant pseudo-Riemannian metric on $M$ is weakly symmetric. We will see additional instances of this in Examples 3.2, 3.3 and 3.4.

Example 3.2. Look at the transitive action of $U(n)$ on $M=S^{2 n-1}(1) \subset \mathbb{C}^{n}$. If $x=(1,0, \cdots, 0)$, then $H_{x}=U(n-1)$. Here $T_{x} M$ can be view as $\operatorname{Im} \mathbb{C}+\mathbb{C}^{n-1}$ and

$$
d g(v, w)=(v, g w)
$$

for any $g \in H_{x}$ and $(v, w) \in \operatorname{Im} \mathbb{C}+\mathbb{C}^{n-1}$. Any $U(n)$-invariant pseudo-Riemannian metric on $M$ is determined by a $U(n-1)$-invariant inner product on the tangent space. Let $\langle,\rangle_{1}$ and $\langle,\rangle_{2}$ be the usual inner products on $\operatorname{Im} \mathbb{C}$ and $\mathbb{C}^{n-1}$ respectively. Then

$$
\left\langle(v, w),\left(v^{\prime}, w^{\prime}\right)\right\rangle=a\left\langle v, v^{\prime}\right\rangle_{1}+b\left\langle w, w^{\prime}\right\rangle_{2}, a \text { and } b \text { nonzero constants, }
$$

is a $U(n-1)$-invariant inner product that induces a $U(n)$-invariant pseudo-Riemannian metric on $M$. The map $\phi$ defined by conjugation on each coordinate is an isometry of $M$. Then $\phi(x)=x$ and $d \phi(v, w)=(-v, \sqrt{-1} w)$ for any $(v, w) \in T_{x} M$. Since $U(n-1)$ acts transitively on the unit sphere in $\mathbb{C}^{n-1}$, it contains an element $g_{w}$ that sends $\sqrt{-1} w$ to $-w$. Hence if $G=U(n) \cup \phi U(n)$, 
then $H_{x}=U(n-1) \cup \phi U(n-1)$, and if $(v, w) \in T_{x} M$ then there exists $k_{w}=g_{w} \cdot \phi \in U(n-1)$ such that

$$
k_{w}(v, w)=(-v,-w)
$$

Hence the $G$-invariant pseudo-Riemannian metric $\langle$,$\rangle on M$ is weakly symmetric, and of course is Riemannian weakly symmetric if $a, b>0$.

Example 3.3. Consider the transitive action of $S p(1) \times S p(n)$ on $M=S^{4 n-1}(1) \subset \mathbb{H}^{n}$ given by $\left(g_{1}, g_{2}\right)(x)=g_{2}(x) g_{1}^{-1}$. The isotropic group of $x=(1,0, \cdots, 0)$ is equal to $\Delta S p(1) \times S p(n-1)$, here $\Delta S p(1)$ means that $S p(1)$ embeds diagonally. The tangent space $T_{x} M$ can be identified with $\operatorname{Im} \mathbb{H}+\mathbb{H}^{n-1}$, and

$$
d\left(g_{1}, g_{2}\right)(v, w)=\left(g_{1} v g_{1}^{-1}, g_{2}(w)\right)
$$

for any $\left(g_{1}, g_{2}\right) \in \Delta S p(1) \times S p(n-1)$. Since $v \mapsto g_{1} v g_{1}^{-1}$ is the standard action of $S p(1) / \mathbb{Z}_{2}=$ $S O(3)$ on $\mathbb{R}^{3}$ and $S p(n-1)$ acts transitively on the unit sphere in $\mathbb{H}^{n-1}$, we have that for any $(v, w) \in T_{x}(M)$ there exists $\left(g_{1}, g_{2}\right) \in \Delta S p(1) \times S p(n-1)$ such that $d\left(g_{1}, g_{2}\right)(v, w)=(-v,-w)$. Hence any $(S p(1) \times S p(n))$-invariant pseudo-Riemannian metric on $M$ is weakly symmetric. Let $\langle,\rangle_{1}$ and $\langle,\rangle_{2}$ be $S p(1)$ - and $S p(n-1)$-invariant metrics on $\operatorname{Im} \mathbb{H}$ and $\mathbb{H}^{n-1}$ respectively. As before, if $a \neq 0 \neq b$ then $a\langle,\rangle_{1}+b\langle,\rangle_{2}$ defines a weakly symmetric $(S p(1) \times S p(n))$-invariant pseudo-Riemannian metric on $M$, which of course is Riemannian if and only if $a, b>0$.

Example 3.4. Let $M=S^{15}=\operatorname{Spin}(9) / \operatorname{Spin}(7)$ with the tangent space $T_{x}(M)=\operatorname{Im} \mathbb{O}+\mathbb{O}$. For any $g \in \operatorname{Spin}(7), d g(v, w)=(\rho(g)(v), \varphi(g)(w))$, where $\rho$ is the standard representation of $\operatorname{Spin}(7)$ on $\operatorname{Im}(1)$ (via the two fold cover $\operatorname{Spin}(7) \rightarrow S O(7)$ ), and where $\varphi$ is the spin representation of $\operatorname{Spin}(7)$ on $\mathbb{O}$. If $(v, w) \in \operatorname{Im} \mathbb{O}+\mathbb{O}$, we have $g_{1} \in \operatorname{Spin}(7)$ such that $\rho\left(g_{1}\right) v=-v$. The isotropy subgroup of $\operatorname{Spin}(7)$ at $v$ (under the action of $\rho$ ) is $\operatorname{Spin}(6)=S U(4)$, and the restriction of the action of $\operatorname{Spin}(7)$ on $\mathbb{O}$ to $S U(4)$ is the standard action of $S U(4)$ on $\mathbb{O}$. That is transitive on the unit sphere, so there exists an element $g_{2} \in \operatorname{Spin}(7)$ such that

$$
\rho\left(g_{2}\right) v=v \text { and } \varphi\left(g_{2}\right) \varphi\left(g_{1}\right) w=-w .
$$

In other words, $d\left(g_{1}, g_{2}\right)(v, w)=(-v,-w)$. Let $\langle,\rangle_{1}$ and $\langle,\rangle_{2}$ be the $\operatorname{Spin}(7)$-invariant inner products on $\operatorname{Im} \mathbb{O}$ and $\mathbb{O}^{n-1}$ respectively. Then $a\langle,\rangle_{1}+b\langle,\rangle_{2}$ is a $\operatorname{Spin}(7)$-invariant inner product on the tangent space of $M=S^{15}$. As before, if $a \neq 0 \neq b$ it induces a weakly symmetric $\operatorname{Spin}(9)$-invariant pseudo-Riemannian metric on $M$. If $a, b>0$ that metric is Riemannian.

\section{Geodesics in PSeudo-Riemannian Weakly symmetric SPaCeS}

In this section we discuss questions of completeness and the geodesic orbit property, and implications for the structure of the nilradical of the isometry group.

Proposition 4.1. Any pseudo-Riemannian weakly symmetric space is geodesically complete.

Proof. It is enough to show that a geodesic $\gamma:[0, a) \rightarrow M$ is extendible. Choose $b$ near $a$ in the interval, let $g$ be the isometry satisfying $g(\gamma(b))=\gamma(b)$ and $g\left(\gamma^{\prime}(b)\right)=-\gamma^{\prime}(b)$. Since $g$ reverses the geodesic through $\gamma(b)$, a reparameterization of $g \circ \gamma$ provides the required extension of $\gamma$.

The main theorem in [1] is that any maximal geodesic in a Riemannian weakly symmetric space $M$ is an orbit of a one-parameter group of isometries of $M$. Proposition 4.1 lets us follow the argument of [1] and push it to the pseudo-Riemannian setting. Thus

Theorem 4.2. Any maximal geodesic in a pseudo-Riemannian weakly symmetric space $M$ is an orbit of a one-parameter group of isometries of $M$.

Definition 4.3. A connected pseudo-Riemannian homogeneous manifold $M$ is said to be a geodesic orbit space if every maximal geodesic in $M$ is an orbit of a one-parameter group of isometries of $M$. 
It is immediate from the definition that a connected pseudo-Riemannian geodesic orbit space is homogeneous.

A connected pseudo-Riemannian manifold which admits a transitive nilpotent group of isometries is said to be a nilmanifold. In the Riemannian case, we have:

Theorem 4.4 ([5]). Let $M$ be a geodesic orbit Riemannian nilmanifold, say with transitive nilpotent group $N$ of isometries. Then $N$ is at most 2-step nilpotent.

By a small extension of Theorem 4.4, for geodesic orbit Riemannian nilmanifolds, we have:

Theorem $4.5([1])$. Let $(M,\langle\rangle$,$) be a connected and simply connected Riemannian geodesic$ orbit space, $G=I(M,\langle,\rangle)^{0}$, and $N$ the nilradical of $G$. Then $N$ is at most 2-step nilpotent.

Combining Theorems 4.4 and 4.5 , we have:

Theorem 4.6. Let $(M,\langle\rangle$,$) be a connected and simply connected Riemannian weakly symmetric$ manifold, $G=I(M,\langle,\rangle)^{0}$, and $N$ the nilradical of $G$. Then $N$ is at most 2-step nilpotent.

This result is due independently to Benson-Ratcliff, Gordon and Vinberg. See [11] for details.

Example 4.7. This is an example of Kath and Olbrich [6, Example 4.6] which shows that Theorem 4.6 fails dramatically in the pseudo-Riemannian case. For each positive integer $m$ define a real Lie algebra $\mathfrak{g}=\mathbb{R}^{m} \oplus \mathbb{C}^{m+1}$. We have the standard real basis $\left\{z_{k}\right\}_{1 \leqq k \leqq m}$ of $\mathbb{R}^{m}$ and the standard complex basis $\left\{e_{i}\right\}_{1 \leqq i \leqq m+1}$ of $\mathbb{C}^{m+1}$, and thus a real basis $\left\{z_{k}\right\} \cup\left\{e_{i}\right\} \cup\left\{f_{j}\right\}$ of $\mathfrak{g}$ where $f_{j}=\sqrt{-1} e_{j}$. The nonzero brackets between the basis vectors are

$$
\left[e_{i}, f_{j}\right]=z_{i+j-1},\left[z_{k}, e_{i}\right]=f_{i+k},\left[z_{k}, f_{j}\right]=-e_{k+j}
$$

where $z_{\ell}=e_{q}=f_{q}=0$ for $\ell>m, q>m+1$. As usual let $\mathfrak{g}=\mathfrak{g}^{0}$ and $\mathfrak{g}^{s+1}=\left[\mathfrak{g}, \mathfrak{g}^{s}\right]$. Then $\mathfrak{g}^{2 r-1}$ is spanned by $\left\{z_{k}, e_{i}, f_{j}\right\}$ with $r+1 \leqq i, j \leqq m+1$ and $r \leqq k \leqq m$ and $\mathfrak{g}^{2 r}$ is spanned by $\left\{z_{k}, e_{i}, f_{j}\right\}$ with $r+1 \leqq i, j \leqq m+1$ and $r+1 \leqq k \leqq m$. Thus $\mathfrak{g}$ is $(2 m+1)$-step nilpotent.

The inner product on $\mathfrak{g}$ is given by $\mathbb{R}^{m} \perp \mathbb{C}^{m+1}$ and

$$
\left\langle e_{i}, f_{j}\right\rangle=0,\left\langle e_{i}, e_{j}\right\rangle=\delta_{i+j, m+2}=\left\langle f_{i}, f_{j}\right\rangle,\left\langle z_{k}, z_{\ell}\right\rangle=\delta_{k+\ell, m+1} .
$$

Consider the corresponding pseudo-Riemannian metric on the connected simply connected nilpotent Lie group $G$ with Lie algebra $\mathfrak{g}$. Compute $\langle x,[y, z]\rangle=\langle[x, y], z\rangle$ to see that the corresponding pseudo-Riemannian metric is bi-invariant. Thus $G$ is a symmetric pseudo-Riemannian nilmanifold which, as Lie group, is $(2 m+1)$-step nilpotent.

We now give a sufficient condition for $N$ to be 2-step nilpotent in the pseudo-Riemannian cases.

Let $\left(M,\langle\right.$,$\rangle be a pseudo-Riemannian homogeneous manifold. Let G \subset I(M)^{0}$ be a closed subgroup that acts transitively on $M$. Let $p \in M$ and $H$ the isotropy group of $G$ at $p$, so $M$ can be identified with $G / H$. The pseudo-Riemannian metric $\langle$,$\rangle on M$ can be viewed as a $G$-invariant metric on $G / H$.

If the metric $\langle$,$\rangle is positive definite, then (G / H,\langle\rangle$,$) is a reductive homogeneous space. If$ the metric $\langle$,$\rangle is indefinite, a reductive decomposition need not exist. See [4] for an example$ of nonreductive pseudo-Riemannian homogeneous space. Fix a fixed reductive decomposition $\mathfrak{g}=\mathfrak{m}+\mathfrak{h}$. We identify $\mathfrak{m} \subset \mathfrak{g}=T_{e} G$ with the tangent space $T_{p} M$ via the projection $\pi$ : $G \rightarrow G / H=M$. Using this identification we view the scalar product $\langle,\rangle_{p}$ on $T_{p} M$ as an $A d(H)$-invariant scalar product on $\mathfrak{m}$.

The definition of a homogeneous geodesic is well-known in the Riemannian case (see, e.g., [7]). In the pseudo-Riemannian case, the necessary generalized version was given in [3]: 
Definition 4.8. Let $M=G / H$ be a pseudo-Riemannian reductive homogeneous space, $\mathfrak{g}=$ $\mathfrak{m}+\mathfrak{h}$ a reductive decomposition, and $p$ the base point of $G / H$. Let $s \mapsto \gamma(s)$ be a geodesic through $p$ with affine parameter $s$ in an open interval $J$. Then $\gamma$ is homogeneous if there exist

1) a diffeomorphism $s=\phi(t)$ between the real line and the open interval $J$ and

2) a vector $X \in \mathfrak{g}$ such that $\gamma(\phi(t))=\exp (t X)(p)$ for all $t \in \mathbb{R}$.

The vector $X$ is then called a geodesic vector.

Remark 4.9. In the Riemannian situation or the pseudo-Riemannian weakly symmetric space cases, the diffeomorphism from the condition 1 is always the identity map on the real line and hence the definition can be formulated more simply.

The basic formula characterizing geodesic vectors in the pseudo-Riemannian case appeared in [4] and [9], but without a proof. The correct mathematical formulation with the proof was given in [3]:

Lemma 4.10. (Geodesic Lemma). Let $M=G / H$ be a reductive pseudo-Riemannian homogeneous space, $\mathfrak{g}=\mathfrak{m}+\mathfrak{h}$ a reductive decomposition and $p$ the base point of $G / H$. Let $X \in \mathfrak{g}$. Then the curve $\gamma(t)=\exp (t X)(p)$ (the orbit of a one-parameter group of isometries) is a geodesic curve with respect to some parameter $s$ if and only if

$$
\left\langle[X, Z]_{\mathfrak{m}}, X_{\mathfrak{m}}\right\rangle=k\left\langle X_{\mathfrak{m}}, Z\right\rangle
$$

for all $Z \in \mathfrak{m}$, where $k \in \mathbb{R}$ is a constant. Further, if $k=0$, then $t$ is an affine parameter for this geodesic. If $k \neq 0$, then $s=e^{-k t}$ is an affine parameter for the geodesic. The second case can occur only if the curve $\gamma(t)$ is a null curve in a (properly) pseudo-Riemannian space.

The following proposition follows easily from the Geodesic Lemma.

Proposition 4.11. Every geodesic in a reductive pseudo-Riemannian homogeneous space $M=$ $G / H$ is an orbit of a one-parameter group of isometries of $G$ if and only if for each $X \in \mathfrak{m}$, there exists $A \in \mathfrak{h}$ such that $\left\langle[X+A, Z]_{\mathfrak{m}}, X\right\rangle=k\langle X, Z\rangle$ for any $Z \in \mathfrak{m}$. In particular, $M$ is a geodesic orbit manifold if and only if this condition holds for $G=I(M)^{0}$.

Now we adapt the argument of [5] to the pseudo-Riemannian case.

Theorem 4.12. Let $(M,\langle\rangle$,$) be a connected pseudo-Riemannian weakly symmetric space, G=$ $I(M,\langle,\rangle)^{0}$, and $N$ the nilradical of $G$. If there is a reductive decomposition $\mathfrak{g}=\mathfrak{m}+\mathfrak{h}$ such that $\mathfrak{n} \subset \mathfrak{m}$ and the metric restriction on $[\mathfrak{n}, \mathfrak{n}]$ is positive or negative definite, then $N$ is at most 2-step nilpotent.

Proof. Let $\mathfrak{a}$ be the orthocomplement of $[\mathfrak{n}, \mathfrak{n}]$ in $\mathfrak{m}$. By Theorem 4.2, $M$ is a geodesic orbit space. Then by Proposition 4.11, for each $X \in \mathfrak{m}$, there exists $A \in \mathfrak{h}$ such that $\left\langle[X+A, Z]_{\mathfrak{m}}, X\right\rangle=$ $k\langle X, Z\rangle$ for any $Z \in \mathfrak{m}$. Fix $\zeta \in \mathfrak{a}$. For any $\xi \in[\mathfrak{n}, \mathfrak{n}]$, there exists $A_{\xi}$ such that

$$
\left\langle\left[\xi+A_{\xi}, \zeta\right]_{\mathfrak{m}}, \xi\right\rangle=k\langle\xi, \zeta\rangle=0 .
$$

Since $\mathfrak{n}$ is an ideal of $\mathfrak{g}$ and $\mathfrak{m}$ is $A d_{G}(H)$-invariant, we have $[\mathfrak{n}, \mathfrak{n}]$ and then $\mathfrak{a}$ are $A d_{G}(H)$ stable. It follows that $\left\langle[\zeta, \xi]_{\mathfrak{m}}, \xi\right\rangle=0$. That is, $\left.\operatorname{ad}(\zeta)\right|_{[\mathfrak{n}, \mathfrak{n}]}$ is skew-symmetric for any $\zeta \in \mathfrak{a}$ by the assumption. But $\operatorname{ad}(\zeta)$ is nilpotent for any $\zeta \in \mathfrak{n}$. So $\operatorname{ad}(\zeta)=0$ for $\zeta \in \mathfrak{n} \cap \mathfrak{a}$. Since $\mathfrak{n} \cap \mathfrak{a}$ generates $\mathfrak{n}$ must have $[\mathfrak{n},[\mathfrak{n}, \mathfrak{n}]]=0$. Thus $\mathfrak{n}$ is at most 2-step nilpotent.

\section{Acknowledgments}

This work is supported by National Natural Science Foundation of China (No.11001133) and the Fundamental Research Funds for the Central Universities. 


\section{REFERENCES}

[1] J. Berndt, O. Kowalski and L. Vanhecke, Geodesics in weakly symmetric spaces, Ann. Global. Anal. Geom., 15(2) (1997), 153-156.

[2] D. Z. Dokovic and N. Q. Thang, Lie groups with dense exponential image, Math. Z. 225 (1997), 35-47.

[3] Z. Dusek and O. Kowalski, Light-like homogeneous geodesics and the geodesic lemma for any signature, Publ. Math. Debrecen. 71/1-2 (2007), 245-252.

[4] J. Figueroa-O'Farrill, P. Meessen and S. Philip, Homogeneity and plane-wave limits, J. High Energy Physics 05 (2005), 050.

[5] C. S. Gordon, Homogeneous Riemannian manifolds whose geodesics are orbits, Topics in geometry, 155-174, Progr. Nonlinear Differential Equations Appl., 20, Birkhäuser Boston, Boston, MA, 1996.

[6] I. Kath and M. Olbrich, The classification problem for pseudo-Riemannian symmetric spaces, examples and applications, 1-52, Recent Developments in Pseudo-Riemannian Geometry, ed. D. V. Alekseevsky 83 H. Baum, ESI Lectures in Mathematics and Physics, European Math. Soc. 2008.

[7] O. Kowalski and L. Vanhecke, Riemannian manifolds with homogeneous geodesics, Boll. Un. Math. Ital. $B$ (7) 5 (1991), 189-246.

[8] B. O'NeIll, Semi-Riemannian geometry with applications to relativity, Pure and Applied Mathematics, 103, Academic Press, Inc. New York, 1983.

[9] S. Philip, Penrose limits of homogeneous spaces, J. Geom. Phys. 56 (2006), 1516-1533.

[10] A. Selberg, Harmonic analysis and discontinuous groups in weakly symmetric spaces with applications to Dirichlet series, J. Indian Math. Soc., 20 (1956), 47-87.

[11] J. A. Wolf, Harmonic analysis on commutative spaces. Mathematical Surveys and Monographs, 142. American Mathematical Society, Providence, RI, 2007.

School of Mathematical Sciences and LPMC, Nankai University, Tianjin 300071, P.R. China

E-mail address: chenzhiqi@nankai.edu.cn

Department of Mathematics, University of California, Berkeley, CA 94720-3840, U.S.A.

E-mail address: jawolf@math.berkeley.edu 